\section{Sur les bases statistiques}

par

S. HARTMAN (Wrocław).

Ce travail est consacré à l'étude de quelques problèmes concernant les propriétés des fonctions et des systèmes de fonctions périodiques et presque périodiques de variable réelle au point de vue de la statistique théorique. Ces problèmes m'ont été suggérés par M. H. Stennhaus; je tiens à lui exprimer ici ma vive gratitude ${ }^{1}$ ).

1. Désignons par $|E|$ la mesure de Lebesgue de l'ensemble $E$ et par $|E|_{R}$ sa mesure relative sur la demi-droite positive, c'est-à-dire le nombre $\lim _{n \rightarrow \infty} 1 / n|E \cdot\langle 0, n\rangle|$.

Définition 1. Appelons distributrice ${ }^{2}$ de la fonction $f(x)$ définie sur la demi-droite $0 \leqslant x<\infty$ et mesurable au sens de Lebesgue la fonction

$$
\varrho(a)=|\underset{x}{E}[f(x)<a]|_{R}
$$

en admettant que la variable a peut prendre les valeurs infinies et en posant

$$
\varrho(-\infty)=0 \quad \text { et } \quad \varrho(+\infty)=1 .
$$

Les fonctions $f(x)$ qui admettent leurs distributrices $\varrho(a)$ pour tout a sont dites relatioement mesurables.

1) Les résultats qui suivent constituent la partie principale de ma Thèse de doctorat, admise par la Faculté de Mathématique, Physique et Chimie de l'Université et École Polytechnique de Wrocław le 15 novembre 1947.

2) Voir par exemple M. Kac et H. Steinhaus, Sur les fonctions indépendantes (IV), (Interballe infini), Studia Mathematica 7 (1938), p. 1. Les distributrices y sont appelées „distribuantes“.
Il existe évidemment des fonctions $f(x)$ définies pour $0 \leqslant x<\infty$ qui sont mesurables au sens de Lebesgue sans être relativement mesurables.

Définition 2. Appelons $\varepsilon$-base statistique de la fonction $f(x)$ définie pour $0 \leqslant x<\infty$ et relativement mesurable tout segment $\langle 0, T(\varepsilon)\rangle$ tel que l'inégalité

$$
\left|\varrho(a)-\frac{|\underset{x}{E}[f(x)<a] \cdot I|}{|I|}\right|<\varepsilon
$$

se présente pour tout a et pour tout segment $I=\langle 0, M\rangle$ où $T(\varepsilon)<M$.

Le plus court des segments $\langle 0, T(\varepsilon)\rangle$ sera dit $\varepsilon$-base statistique minimum et sa longueur sera désignée par $T_{\min }(\varepsilon)$.

On peut évidemment remplacer dans cette définition les segments par les intervalles ouverts ou semi-ouverts ayant les mêmes extrémités.

Il existe des fonctions relativement mesurables qui n'admettent pas de $\varepsilon$-base statistique pour tout $\varepsilon>0$. Ainsi, par exemple, la fonction $f(x)=1-1 / x$ n'en admet pour aucun $\varepsilon<1$. En effet, on a $\varrho(a)=0$ pour tout $a<1$, tandis que $1 / n|\underset{x}{E}[f(x)<a] \cdot\langle 0, n\rangle|=1$ pour $a>1-1 / n$. ll est aisé de montrer que la condition nécessaire et suffisante pour que $f(x)$ admette une $\varepsilon$-base statistique pour tout $\varepsilon>0$ est la convergence uniforme de la suite des fonctions

$$
F_{n}(a)=1 / n\left|E_{x}[f(x)<a] \cdot\langle 0, n\rangle\right|
$$

que M. MarczewsKi et $\mathrm{M}^{\text {Mle }}$ NosarzewsKa appellent distributrices approchées ${ }^{3}$ ) de $f(x)$. Les mêmes auteurs ont montré ${ }^{4}$ ) que la condition suffisante pour la convergence uniforme de la suite $\left\{F_{n}(a)\right\}$ dans tous les intervalles finis est la continuité de la distributrice $\rho(a)$. C'est donc également une condition suffisante pour qu'une fonction bornée $f(x)$ admette une $\varepsilon$-base statistique pour tout $\varepsilon>0$.

\footnotetext{
3) E. Marczewski et M. Nosarzewska, Sur la convergence uniforme et la mesurabilité relative, Colloquium Mathematicum I.1 (1947), p. 15-18.
}

4) Ibidem, p. 18 
Les considérations ultérieures sur les bases statistiques seront bornées aux fonctions presque périodiques au sens de BoHR ${ }^{5}$ ), qui constituent une classe typique de fonctions $f(x)$ dont les distributrices $\varrho(a)$ sont définies pour tout a sauf tout au plus pour une infinité dénombrable de valeurs ${ }^{6}$ ) et - dans les cas les plus fréquents - pour toutes les valeurs de a (les considérations analogues pour les bases statistiques des fonctions périodiques étant triviales). Nous n'envisagerons d'ailleurs que les fonctions presque périodiques dont les distributrices sont définies pour tout $a$ et continues, donc qui admettent leurs $\varepsilon$-bases statistiques pour tout $\varepsilon>0$. Cette hypothèse est satisfaite en particulier ${ }^{7}$ ) par les fonctions presque périodiques dont les fréquences ${ }^{8}$ ) sont linéairement indépendantes.

Si la distributrice d'une fonction est continue, elle est uniformément continue sur le segment (fermé) infini $\langle 0,+\infty\rangle$, car elle y est bornée et monotone.

Définition 5. Appelons $\varepsilon$-module local de continuité de la fonction continue $f(x)$ au point $x_{0}$ - et désignons-le par $\delta_{f}\left(x_{0}, \varepsilon\right)-$ le plus grand des nombres $\delta$. pour lesquels $\left|x-x_{0}\right|<\delta$ entraîne $\left|f(x)-f\left(x_{0}\right)\right|<\varepsilon$ (bien entendu, $\varepsilon$ est supposé positif).

Définition 4. Appelons $\varepsilon$-module de continuité uniforme de la fonction $f(x)$ uniformément continue dans l'intervalle infini $(-\infty,+\infty)$ - et désignons ce module par $\delta_{f}(\varepsilon)-$ le plus grand des nombres $\delta$ tels que $\left|x-x_{0}\right|<\delta$ entraîne $\left|f(x)-f\left(x_{0}\right)\right|<\varepsilon$ pour tout $x_{0}$ (où encore $\varepsilon>0$ ).

Soit $f(x)$ une fonction presque périodique au sens de Bонr. Pour tout $\eta>0$, il existe alors par définition un $L(\eta)>0$ tel que tout segment de longueur $L(\eta)$ contient une $\eta$-presque période

5) H. Bohr, Fastperiodische Funktionen, Ergebnisse der Mathematik 1 (1932), p. $27-28$

6) A. Wintner, Über die statistische Unabhängigkeit der asymptotischen Verteilungsfunktionen inkommensurabler Partialschwingungen, Mathematische Zeitschrift 36 (1933), p. 618-629.

7) A. Wintn er, Über die Stetigkeit der asymptotischen Verteilungsfunktion bei inkommensurablen Partialschringungen, Mathematische Zeitschrift 37 (1933), p. 279-280.

8) H. Bohr, op cit., p. 45. Les fréquences y sont appelées „Fourierexponenten:c. $\tau(\eta)$, c'est-à-dire un $\tau(\eta)$ satisfaisant pour tout $x$ à l'inégalité $|f(x+\tau(\eta))-f(x)|<\eta$. On exprime cette propriété de $f(x)$ tout court en disant que, pour tout $\eta>0$, l'ensemble des $\eta$-presque périodes $\tau(\eta)$ est relatioement dense.

Théor ème I. Si la fonction $f(x)$ est presque périodique et sa distributrice $\varrho$ (a) est continue en tout $a$, on a pour tout $\varepsilon>0$ l'estimation suivante de sa $\varepsilon$-base statistique:

$$
T_{\min }(\varepsilon) \leqslant 5 / \varepsilon L\left(\delta_{e}\left(\varepsilon^{2} / 5\right)\right) \text {. }
$$

Démonstration. Il existe par hypothèse la limite

$$
\varrho(a)=\lim _{T \rightarrow \infty} \frac{1}{T}|\underset{x}{E}[f(x)<a ; x \leqslant T]|
$$

quel que soit a. Soit $\varepsilon>0$. Posons

$$
\eta=\delta_{\rho}\left({ }^{e} / 5\right)
$$

et choisissons un $T>5 L(\eta) / \varepsilon$. Il s'agit de montrer que

$$
\left|\frac{1}{T}\right| \underset{x}{E}[f(x)<a ; x \leqslant T]|-\varrho(a)|<\varepsilon .
$$

Considérons le segment $I_{1}=\langle 0, T\rangle$ de l'axe d'abscisses; déplaçons-le de $\tau_{1}=\inf \tau(\eta)$ à droite et désignons par $I_{2}$ le segment égal à $I_{1}$, ainsi obtenu. Déplaçons ensuite $I_{1}$ de $\underset{\tau_{2}}{\tau_{\tau(\eta) \geqslant T+\tau_{1}}} \underset{\text { inf }}{ } \tau(\eta)$ droite et désignons par $I_{3}$ le segment obtenu. De façon générale: $I_{n}$ désignera le segment qui s'obtient de $I_{1}$ en le déplaçant à droite de $\tau_{n-1}=\inf _{\tau(\eta) \gg T+\tau_{n-2}} \tau(\eta)$ où $\tau_{0}=0$.

Les termes successifs de la suite $\left\{I_{n}\right\}$ sont donc des segments distants tout au plus de $L=L(\eta)$ l'un de l'autre. Par conséquent:

$$
0 \leqslant \tau_{n+1}-\left(T+\tau_{n}\right) \leqslant L .
$$

On a $\left|f\left(x+\tau_{n}\right)-f(x)\right| \leqslant \eta$ pour tout $x$ et $f(x)<a-\eta$ entraîne $f\left(x+\tau_{n}\right)<a$. Par conséquent:

$$
E_{x}\left[f\left(x-\tau_{n-1}\right)<a-\eta\right] \cdot I_{n} \subset \underset{x}{E}[f(x)<a] \cdot I_{n} .
$$


Désignons pour abréger par $E_{n}$ le membre droit et par $E_{n}^{*}$ le membre gauche de cette inclusion. On a donc $E_{n}^{*} \subset E_{n}$ pour $n=1,2, \ldots$, et tout ensemble $E_{n}^{*}$ est superposable à l'ensemble

$$
E_{1}^{*}=E_{x}[f(x)<a-\eta] \cdot I_{1} .
$$

Soient enfin $M=\left|E_{n}^{*}\right|$ et $C-$ le complémentaire de l'ensemble $\sum_{n=1}^{\infty} I_{n}$. II vient:

(5) $\quad \sum_{n=1}^{\infty} E_{n}=\sum_{n=1}^{\infty} E_{n}^{*}+\sum_{n=1}^{\infty} E[f(x)<a-\eta] \cdot\left[I_{n}-E_{n}^{*}\right]+$

$$
+\sum_{n=1}^{\infty} E[a-\eta \leqslant f(x)<a] \cdot\left[I_{n}-E_{n}^{*}\right]
$$

$$
E_{x}[f(x)<a]=\sum_{n=1}^{\infty} E_{n}+C \cdot E_{x}[f(x)<a] .
$$

Nous allons estimer les mesures relatives supérieures ${ }^{9}$ ) des trois sommes constituant le membre droit de l'égalité (5).

$1^{0}$ On a en vertu de (4)

$$
\frac{M}{T+L} \leqslant\left|\sum_{n=1}^{\infty} E_{n}^{*}\right|_{\mid \bar{R}} \leqslant \frac{M}{T}
$$

mais on notera aussitôt, en tenant compte du choix de $T$, que

$$
\frac{M}{T}-\frac{M}{L+T}=\frac{M L}{T(L+T)}<\frac{L}{T}<\frac{\varepsilon}{5} .
$$

$2^{0}$ En désignant par $A_{n}$ le $n$-ième sommande de la deuxième somme figurant dans le membre droit de (5), $x \in A_{n}$ entraîne $a-\eta \leqslant f\left(x-\tau_{n-1}\right)$, d'où $a-2 \eta \leqslant f(x)<a-\eta$ et

$$
\sum_{n=1}^{\infty} A_{n} \subset E_{x}[a-2 \eta \leqslant f(x)<a-\eta]
$$

mais on a en vertu de (2)

$$
|\underset{x}{E}[a-2 \eta \leqslant f(x)<a-\eta]|_{R}=\varrho(a-\eta)-\varrho(a-2 \eta) \leqslant \frac{\varepsilon}{5} .
$$

Il en résulte que $\left|\sum_{n=1}^{\infty} A_{n}\right|_{\bar{R}} \leqslant \frac{\varepsilon}{5}$.

9) La mesure relatioe supérieure sur la demi-droite positive d'un ensemble $E$ mesurable au sens de Lebesgue est définie par la formule

$$
|E|_{\bar{R}}=\limsup _{n \rightarrow \infty} \frac{1}{n}|E \cdot\langle 0, n\rangle| \text {. }
$$

La définition de sa mesure relative inférieure $|E|_{\underline{R}}$ est analogue.
$3^{0}$ La troisième somme est contenue dans $\underset{x}{E}[a-\eta \leqslant f(x)<a]$; mais on a encore d'après (2)

$$
|\underset{x}{E}[a-\eta \leqslant f(x)<a]|_{R}=\varrho(a)-\varrho(a-\eta) \leqslant \frac{\varepsilon}{5},
$$

d'où

$$
\left|\sum_{n=1}^{\infty} E[a-\eta \leqslant f(x)<a] \cdot\left[I_{n}-E_{n}^{*}\right]\right|_{\bar{R}} \leqslant \frac{\varepsilon}{5} .
$$

En vertu des formules (5)-(z) et des estimations $1^{0}-3^{0}$, on peut écrire, en tenant compte de l'estimation évidente $|C|_{\bar{k}} \leqslant L / T<<^{8 / 5}$ :

$$
\frac{M}{T+L} \leqslant \varrho(a)<\frac{M}{T+L}+\frac{\varepsilon}{5}+\frac{\varepsilon}{5}+\frac{\varepsilon}{5}+\frac{\varepsilon}{5}=\frac{M}{T+L}+\frac{4 \varepsilon}{5} .
$$

On a d'autre part

$$
\underset{x}{E}[f(x)<a] \cdot I_{1}=E_{1}^{*}+\underset{x}{E}[a-\eta \leqslant f(x)<a] \cdot I_{1} .
$$

En désignant par $B$ le dernier sommande de cette formule et par $B\left(\tau_{n}\right)$ le même ensemble déplacé de $\tau_{n}$ à droite, $x \in B\left(\tau_{n}\right)$ entraîne $x-\tau_{n} \in B$, d'où $a-2 \eta \leqslant f(x)<a+\eta$. Il en résulte (en posant $\left.B=B\left(\tau_{0}\right)\right)$ que

$$
\sum_{n=0}^{\infty} B\left(\tau_{n}\right) \subset E_{x}[a-2 \eta \leqslant f(x)<a+\eta] \text {, d'où }\left|\sum_{n=0}^{\infty} B\left(\tau_{n}\right)\right|_{\bar{n}} \leqslant \frac{3 \varepsilon}{5}
$$

en vertu de (2). Soit $\mu=|B|$. Il vient:

$$
\left|\sum_{n=0}^{\infty} B\left(\tau_{n}\right)\right| \geqslant \limsup _{n \rightarrow \infty} \frac{\sum_{k=0}^{n} B\left(\tau_{k}\right)}{T+\tau_{n}} \geqslant \limsup _{n \rightarrow \infty} \frac{\mu \cdot(n+1)}{T \cdot(n+1)+L \cdot n}=\frac{\mu}{T+L},
$$

d'où

On a aussi

$$
\frac{\mu}{T+L} \leqslant \frac{3 \varepsilon}{5}
$$

$$
\frac{1}{T}\left|E_{x}[f(x)<\mathrm{a}] \cdot I_{1}\right|=\frac{M+\mu}{T} .
$$

Comme $\frac{\mu}{T}<\frac{\mu+L}{T+L}$, puisque $\mu<T$, et $\frac{\mu+L}{T+L}<\frac{\mu}{T}+\frac{L}{T}$, il vient en vertu de (9) et en tenant compte du choix de $T$ :

$$
\frac{\mu}{T}<\frac{3 \varepsilon}{5}+\frac{\varepsilon}{5}=\frac{4 \varepsilon}{5}
$$


On a enfin en vertu de (7) et (11)

$$
\frac{M+\mu}{T}-\frac{M}{L+T}<\frac{4 \varepsilon}{5}+\frac{\varepsilon}{5}=\varepsilon .
$$

On conclut de (8), (10) et (12) que

$$
\left|\frac{1}{T}\right| E_{x}[f(x)<a] \cdot I_{1}|-\varrho(a)|<\varepsilon
$$

on a donc la formule (3), c. q. f. d.

2. Définition 1'. Appelons distributrice du système $F$ de fonctions $f_{1}(x), \ldots, f_{m}(x)$ définies pour $0 \leqslant x<\infty$ et mesurables au sens de Lebesgue la fonction de $m$ variables

$$
\varrho\left(a_{1}, \ldots, a_{m}\right)=\mid E\left[f_{1}(x)<a_{1}, \ldots, f_{m}(x)<a_{m}\right]_{R} .
$$

Les fonctions qui forment le système $F$ dont la distributrice est définie pour tout système de nombres $a_{1}, \ldots, a_{m}$ seront dites relatioement mesurables dans leur ensemble.

Définition $2^{\prime}$. Appelons $\varepsilon$-base statistique du système $F$ de fonctions $f_{1}(x), \ldots, f_{m}(x)$ définies pour $0 \leqslant x<\infty$ et relativement mesurables dans leur ensemble tout segment $\langle 0, T(F, \varepsilon)\rangle$ tel que l'inégalité

$$
\text { (1') }\left|\varrho\left(a_{1}, \ldots, a_{m}\right)-\frac{\left|E_{x}\left[f_{1}(x)<a_{1}, \ldots ., f_{m}(x)<a_{m}\right] \cdot I\right|}{|I|}\right|<\varepsilon
$$

se présente pour tout système de nombres $a_{1}, \ldots, a_{m}$ et pour tout segment $I=\langle 0, M\rangle$ où $T(F, \varepsilon)<M$.

Le plus court des segments $\langle 0, T(F, \varepsilon)\rangle$ sera dit $\varepsilon$-base statistique minimum du système $F$ et sa longueur sera désignée par $T_{\min }(F, \varepsilon)$.

Rappelons que les fonctions formant le système $F$ s'appellent statistiquement (ou stochastiquement) indépendantes $\left.{ }^{10}\right)$, lorsqu'on

${ }^{10}$ ) selon M. Kac et H. Steinhaus, loco cit., p. 3-4. On y exige davantage, à savoir que la condition

$$
\left|E_{x}\left[f_{1}(x) \in I_{1}, \ldots, f_{m}(x) \in I_{m}\right]\right|_{R}=\prod_{i=1}^{m}\left|E_{x}\left[f_{i}(x) \in I_{i}\right]\right|_{R}
$$

soit satisfaite pour tout système d'intervalles $I_{1}, \ldots, I_{m}$ ouverts, demi-ouverts ou fermés. Or, cette condition revient à (13) lorsqu'on a pour tout a (où $i=1,2, \ldots, m$ ) a pour tout système de valeurs (même infinies) de $a_{1}, \ldots, a_{m}$

$$
\varrho\left(a_{1}, \ldots, a_{m}\right)=\prod_{i=1}^{m} \varrho_{i}\left(a_{i}\right),
$$

où $\varrho$ désigne la distributrice du système $F$ et $\varrho_{i}$ - celle de la fonction $f_{i}$ de ce système.

Nous allons considérer dans la suite les systèmes $F$ de fonctions presque périodiques. Pour tout $\eta>0$, il existe dans leur cas ${ }^{11}$ ) un ensemble relativement dense de $\eta$-presque périodes communes du système $F$, c'est-à-dire qu'il existe un $L(F, \eta)>0$ tel que tout segment de longueur $L(F, \eta)$ contient un $\tau(F, \eta)$ satisfaisant à l'inégalité $\left|f_{i}(x+\tau(F, \eta))-f_{i}(x)\right|<\eta$ pour tout $x$ et pour tout $i=1, \ldots, m$.

Nous allons nous borner aux systèmes $F$ de fonctions presque périodiques, relativement mesurables dans leur ensemble et dont les distributrices sont continues. Ces hypothèses sont satisfaites en particulier lorsque toutes les fréquences de n'importe quelle fonction du système $F$, envisagées dans leur ensemble, sont linéairement indépendantes. Alors - comme on sait ${ }^{12}$ ) ces fonctions sont statistiquement indépendantes.

Notons à ce propos que si un tel système $F$ de fonctions statistiquement indépendantes admet pour tout $\varepsilon>0$ une $\varepsilon$-base statistique susceptible d'estimation, on peut déterminer aisément un segment $\langle 0, T(F, \varepsilon)\rangle$ tel que l'inégalité

$$
\left|\frac{1}{|I|^{m}} \prod_{i=1}^{m}\right| E_{x}\left[f_{i}(x)<a_{i}\right] \cdot I\left|\frac{1}{|I|}\right| \prod_{i=1}^{m} E_{x}\left[f_{i}(x)<a_{i}\right] \cdot I||<\varepsilon
$$

se présente pour tout système de nombres $a_{1}, \ldots, a_{m}$ et pour tout segment $I=\langle 0, M\rangle$ où $T(F, \varepsilon)<M$. Or, l'inégalité (14) peut être regardée comme une approximation de l'inégalité (15) qui définit la notion d'indépendance statistique.

l'égalité $\left.\underset{x}{\mid}\left[f_{i}(x)=a\right]\right|_{R}=0$, qui se présente en particulier quand les distributrices $\varrho_{i}(a)$ sont continues - ce que nous avons précisément admis pour les fonctions dont il s'agit dans ce travail (voir p. 122).

11) H. Bohr, op. cit., p. 31-32.

12) Voir par exemple F. Wecken, Abstrakte Integrale und fastperiodische Funktionen, Mathematische Zeitschrift 45 (1939), p. 402 
Théor ème I'. Lorsque les fonctions presque périodiques $f_{1}(x), \ldots, f_{m}(x)$ sont relatidement mesurables dans leur ensemble et leurs distributrices $\varrho_{1}(a), \ldots, \varrho_{m}(a)$ sont continues en tout $a$, le système $F$ de ces fonctions admet pour tout $\varepsilon>0$ une $\varepsilon$-base statistique et on a l'estimation suioante:

$$
T_{\min }(F, \varepsilon) \leqslant 5 / \varepsilon \cdot L\left(F, \min _{i=1, \ldots, m} \delta_{\rho_{i}}\left({ }^{8} / 5 m\right)\right) .
$$

Le théorème I est un cas particulier du théorème I' (en posant $m=1$ ), mais la démonstration pour le système de plusieurs fonctions étant plus compliquée, quoique analogue, il a été préférable de la faire précéder par une plus simple.

Démonstration. Posons:

$$
\eta=\min _{i=1, \ldots, m} \delta_{\rho_{i}}(\varepsilon / 5 m), \quad L=L(F, \eta)
$$

et choisissons un $T>5 L / \varepsilon$.

Construisons, tout comme dans la démonstration du théorème $I$, la suite de segments $\left\{I_{n}\right\}$, en déplaçant le segment $\langle 0, T\rangle$ successivement de $\tau_{n-1}=\inf _{\tau(\eta) \geqslant T+\tau_{n-2}} \tau(\eta)$ pour $n=0,1,2, \ldots$, où $\tau_{0}=0$ et $\tau(\eta)$ désignent à présent des $\eta$-presque périodes communes des fonctions $f_{1}, \ldots, f_{m}$.

$E_{n}$ désignera maintenant l'ensemble

et $E_{n}^{*}-$ l'ensemble

$$
\underset{x}{E}\left[f_{1}(x)<a_{1}, \ldots, f_{m}(x)<a_{m}\right] \cdot I_{n}
$$

$$
E_{x}\left[f_{1}\left(x-\tau_{n-1}\right)<a_{1}-\eta, \ldots, f_{m}\left(x-\tau_{n-1}\right)<a_{m}-\eta\right] \cdot I_{n} .
$$

Soit enfin, comme auparavant, $M=\left|E_{n}^{*}\right|$ et $C=$ complémentaire de $\sum_{n=1}^{\infty} I_{n}$. Au lieu de (5) et (6), nous aurons à présent:

$$
\begin{gathered}
\sum_{n=1}^{\infty} E_{n}^{*} \subset \sum_{n=1}^{\infty} E_{n} \subset \sum_{n=1}^{\infty} E_{n}^{*}+ \\
+\sum_{n=1}^{\infty} E\left[f_{1}(x)<a_{1}-\eta, \ldots, f_{m}(x)<a_{m}-\eta\right] \cdot\left[I_{n}-E_{n}^{*}\right]+ \\
+\sum_{n=1}^{\infty} \sum_{i=1}^{m} E_{x}\left[a_{i}-\eta \leqslant f_{i}(x)<a_{i}\right] \cdot\left[I_{n}-E_{n}^{*}\right]
\end{gathered}
$$

(6') $\underset{x}{E}\left[f_{1}(x)<a_{1}, \ldots, f_{m}(x)<a_{m}\right]=\sum_{n=1}^{\infty} E_{n}+C \cdot \underset{x}{E}\left[f_{1}(x)<a_{1}, \ldots, f_{m}(x)<a_{m}\right]$.
L'estimation $1^{0}$ et l'inégalité (7) restent les mêmes, mais on a, au lieu de $2^{0}$, l'estimation suivante:

$2^{0^{\prime}}$ En désignant par $A_{n}$ le $n$-ième sommande de la deuxième somme figurant dans le membre droit de $\left(5^{\prime}\right), x \in A_{n}$ entraîne $a_{i}-\eta \leqslant f_{i}\left(x-\tau_{n-1}\right)<a_{i}$ pour l'une au moins des valeurs $i=1, \ldots, m$; il en résulte $a_{i}-2 \eta \leqslant f_{i}(x)<a_{i}-\eta$ pour la même valeur de $i$, d'où

$$
\sum_{n=1}^{\infty} A_{n} \subset \sum_{i=1}^{m} E\left[a_{i}-2 \eta \leqslant f_{i}(x)<a_{i}-\eta\right]
$$

mais comme

$$
\left|E_{x}\left[a_{i}-2 \eta \leqslant f_{i}(x)<a_{i}-\eta\right]\right|_{R}=\varrho_{i}\left(a_{i}-\eta\right)-\varrho_{i}\left(a_{i}-2 \eta\right),
$$

il vient en vertu de $\left(2^{\prime}\right)$

$$
\left|\sum_{n=1}^{\infty} A_{n}\right|_{\bar{R}} \leqslant \frac{\varepsilon}{5 m} \cdot m=\frac{\varepsilon}{5} .
$$

L'estimation $3^{0}$ est à remplacer par

$3^{0^{\prime}}$ La troisième somme (double) figurant dans le membre droit de $\left(5^{\prime}\right)$ est contenue dans $\sum_{i=1}^{m} E\left[a_{i}-\eta \leqslant f_{i}(x)<a_{i}\right]$; par conséquent, sa mesure relative supérieure ne dépasse pas $m \varepsilon / 5 m=8 / 5$.

En tenant compte de l'estimation évidente $|C|_{\bar{R}} \leqslant L / T<\varepsilon / 5$, on obtient au lieu de (8)

$$
\frac{M}{T+L} \leqslant \varrho\left(a_{1}, \ldots, a_{m}\right)<\frac{M}{T+L}+\frac{4 \varepsilon}{5} .
$$

En posant

$$
B=\sum_{i=1}^{m} E_{x}\left[a_{i}-\eta \leqslant f_{i}(x)<a_{i}\right] \cdot I_{1}
$$

et en désignant par $B\left(\tau_{n}\right)$ le même ensemble déplacé de $\tau_{n}$ vers la droite, $x \in B\left(\tau_{n}\right)$ entraîne $a_{i}-\eta \leqslant f_{i}\left(x-\tau_{n}\right)<a_{i}$ pour l'une au moins des valeurs de $i=1, \ldots, m$; on a par conséquent

$$
a_{i}-2 \eta \leqslant f_{i}(x)<a_{i}+\eta
$$

pour la même valeur de $i$, d'où (en posant $B=B\left(\tau_{0}\right)$ )

$$
\sum_{n=0}^{\infty} B\left(\tau_{n}\right) \subset \sum_{i=1}^{m} E_{x}\left[a_{i}-2 \eta \leqslant f_{i}(x)<a_{i}+\eta\right]
$$

Studia Mathematica. T. X. 
Il en résulte d'après ( $\left(2^{\prime}\right)$

$$
\left|\sum_{n=0}^{\infty} B\left(\tau_{n}\right)\right|_{\bar{R}} \leqslant m \cdot \frac{3 \varepsilon}{5 m}=\frac{3 \varepsilon}{5},
$$

de. sorte qu'en posant $\mu=|B|$, l'inégalité (9) garde sa validité. .

Comme

$$
E_{1}^{*} \subset E_{x}\left[f_{1}(x)<a_{1}, \ldots, f_{m}(x)<a_{m}\right] \cdot l_{1} \subset E_{1}^{*}+B,
$$

on a au lieu de (10)

$$
\frac{M}{T} \leqslant \frac{1}{T}\left|E_{x}\left[f_{1}(x)<a_{1}, \ldots, f_{m}(x)<a_{m}\right] \cdot I_{1}\right| \leqslant \frac{M+\mu}{T} .
$$

Enfin, l'inégalité (11) subsiste et on conclut de $(7),\left(8^{\prime}\right),\left(10^{\prime}\right)$ et (11) que

$$
\left|\frac{1}{T}\right| \underset{x}{E}\left[f_{1}(x)<a_{1}, \ldots, f_{m}(x)<a_{m}\right] \cdot I_{1}\left|-\varrho\left(a_{1}, \ldots, a_{m}\right)\right|<\varepsilon, \quad \text { c. q. f. d. }
$$

On remarquera que l'hypothèse d'après laquelle le point initial de l'intervalle $L$ était au zéro n'intervenait nulle part dans les démonstrations des théorèmes $I$ et $I^{\prime}$. Il en résulte que n'importe quel intervalle de longueur $T$ peut remplacer $I$ dans les formules (1) et $\left(1^{\prime}\right)$ des définitions 2 et $2^{\prime}$ respectivement. On voit ainsi que les bases statistiques d'une fonction presque périodique à distributrice continue ou d'un système de telles fonctions relativement mesurables dans leur ensemble se laissent déplacer arbitrairement.

Or, cette propriété des bases statistiques ne subsiste guère pour une fonction quelconque admettant des $\varepsilon$-bases statistiques pour tous les $\varepsilon>0$. En effet, il suffit de placer dans les intervalles successifs de longueur $1,2, \ldots$ à partir du point 0 des ondes sinusoïdales uniques ayant la même amplitude, pour obtenir une fonction dont la distributrice est d'ailleurs continue et qui admet pour tout $\varepsilon>0$ une $\varepsilon$-base statistique (au sens de la définition 2), tout en cessant de satisfaire à la relation (1) pour des intervalles $I$ déplacés suffisamment loin vers la droite.

3. La grandeur $\delta_{e}(e / 5)$ ou $\min _{i=1, \ldots, m} \delta_{e_{i}}(e / 5 m)$, à savoir le module de continuité uniforme, qui figure dans les thèses du théorème I et du théorème $I^{\prime}$ respectivement peut être facilement déterminée ou estimée inférieurement dans des cas simples. Cette estimation suffit parfaitement, car on peut évidemment remplacer dans les thèses en question, au prix de les rendre moins précises, le module de continuité uniforme par un nombre plus petit quelconque.

Voici quelques exemples de l'évaluation et de l'estimation de $\delta_{\rho}(\varepsilon)$ :

1. $f(x)=A \cos \lambda x$ ou $A \sin \lambda x$, On a pour $\varepsilon<1$

$$
\delta_{\rho}(\varepsilon)=2 A \sin ^{2} \frac{\varepsilon \pi}{2} \text {. }
$$

En effet, les valeurs de a pour lesquelles le module local de continuité de la distributrice $\varrho(a)$ est pour tout $\varepsilon>0$ minimum, donc égal à celui de continuité uniforme, sont égales dans le cas considéré à $A$ et à $-\boldsymbol{A}$ respectivement. Il s'agit par conséquent de trouver ce module local.

Soit $\vartheta$ un nombre tel que $A \cos \lambda \vartheta=A-\delta_{\varphi}(\varepsilon)$ et admettons que la mesure relative de l'image réciproque de l'intervalle $\left(A-\delta_{\varrho}(\varepsilon), A\right)$, égale à l'accroissement $\varrho(A)-\varrho\left(A-\delta_{\rho}(\varepsilon)\right)$ de la distributrice, soit égale à $\varepsilon$. Il vient alors

$$
\frac{\vartheta}{\pi} \lambda=\varepsilon, \quad \text { d'où } \quad \delta_{\rho}(\varepsilon)=A\left(1-\cos \lambda \frac{\varepsilon \pi}{\lambda}\right)
$$

et par suite l'égalité (15).

Dans ce cas, $\delta_{\rho}(\varepsilon)$ ne dépend donc pas de la fréquence $\lambda$.

2. $f(x)=\sum_{k=1}^{m} \psi_{k}(x)$ où $\psi_{k}(x)=A_{k} \cos \lambda_{k} x+B_{k} \sin \lambda_{k}$. Si les $\lambda_{k}$ sont linéairement indépendants, les $\psi_{k}$ le sont statistiquement, de même que toute somme partielle est statistiquement indépendante de chacun des autres sommandes $\left.{ }^{13}\right)$.

Désignons par $\sigma(t), \sigma_{k}(t)$ et $\varrho_{k}(t)$ respectivement les distributrices des fonctions $f(x), \sum_{i=1}^{k} \psi_{i}(x)$ où $k=1,2, \ldots, m$ et de $\psi_{k}(x)$. On peut évaluer de proche en proche $\sigma(t)$ par intégrales de Stieltjes (produits de composition) au moyen de la formule recurrente suivante:

$$
\begin{gathered}
\sigma_{k}(t)=\int_{-\infty}^{\infty} \varrho_{k}(t-\xi) d \sigma_{k-1}(\xi), \quad k=1,2, \ldots, m-1, \\
\sigma_{m}(t)=\sigma(t) .
\end{gathered}
$$

13) A. Wintner, loco cit., Mathematische Zeitschrift 36. 
Il résulte de cette formule que

$$
\begin{array}{r}
\left|\sigma_{k}(t+\Delta t)-\sigma_{k}(t)\right| \leqslant \int_{-\infty}^{\infty}\left|\varrho_{k}(t+\Delta t-\xi)-\varrho_{k}(t-\xi)\right| d \sigma_{k-1}(\xi) \leqslant \\
\cdot \quad \leqslant \max \left|\varrho_{k}(t+\Delta t)-\varrho_{k}(t)\right|,
\end{array}
$$

$\operatorname{car} \int^{\infty} d \sigma_{k-1}(\xi)=1$. On a donc pour tout $\varepsilon>0$

$$
\delta_{e_{k}}(\varepsilon) \leqslant \delta_{\sigma}(\varepsilon), \quad k=1,2, \ldots, m .
$$

3. Le même raisonnement est applicable à la somme de fonctions périodiques quelconques ayant les fréquences linéairement indépendantes. En vertu de l'inégalité (16), on peut donc dans ces cas substituer à $\delta_{\rho}\left({ }^{(8 / 5)}\right.$ le module de continuité uniforme de la distributrice du sommande quelconque. Pour avoir une estimation aussi précise que possible de la $\varepsilon$-base statistique, il faut prendre le plus grand de ces modules.

Ce n'est que l'estimation supérieure de $L(\eta)$ pour la valeur donnée de $\eta$ qui comporte des difficultés.

Dans le cas où $f(x)$ est somme de deux fonctions périodiques aux périodes (donc aussi aux fréquences) incommensurables, nous allons établir un algorithme fini qui conduit à la solution de ce problème. Dans des cas plus particuliers, l'estimation de $L(\eta)$ pourra se faire même à l'aide d'une formule directe (cf. Théorème III, p. 136, et son application, p. 138).

4. Rappelons d'abord un théorème appartenant à la théorie des approximations diophantiques, à savoir le théorème de HECKE dans sa forme généralisée par Ostrowski ${ }^{14}$ ).

Désignons par $R(x)$ le reste $\bmod 1$ du nombre $x$ :

$$
R(x)=x-\mathrm{E} x .
$$

Soit $\vartheta$ un nombre réel quelconque dans l'intervalle $(\alpha, \beta)$ situé entre 0 et 1 . Désignons, pour tout $N$ naturel, par $N^{\prime}$ le nombre des $k \leqslant N$ naturels tels que

$$
\alpha \leqslant R(k \vartheta)<\beta .
$$

14) A. Ostrowski, Mathematische Miszellen XVI, Zur Theorie der linearen diophantischen Approximationen, Jahresberichte der Deutschen Math. Verein: 39 (1930), p. 34-46.
On a alors le théorème suivant (de Hecke-Ostrowsri):

Si $\beta-\alpha=R(\vartheta q)$ où $\vartheta$ est un nombre réel donné et $q$ est un nombre naturel, on a $\left|N^{\prime}-\bar{N} \cdot R(q \vartheta)\right|<q$ pour tout $N$ naturel.

Il en résulte aussitôt que la fréquence des nombres naturels ${ }^{15}$ ) $k$ satisfaisant à (17) est égale à $\beta-\alpha$ (en accord avec le théorème de $\mathrm{H}$. WeYL établi pour $\vartheta$ irrationnel).

Passons à l'estimation de $L(F, \eta)$ pour le système $F$ composé de deux fonctions périodiques et continues, $f_{1}$ et $f_{2}$, dont les périodes sont incommensurables (autrement, le problème étant trivial).

Remarque. On peut en passer aussitôt à l'estimation de $L\left(f_{1}+f_{2}, \eta\right)$, c'est-à-dire de la grandeur $L(\eta)$ pour la fonction $f_{1}+f_{2}$ :

$$
L\left(f_{1}+f_{2}, \eta\right) \leqslant L(F, \eta / 2),
$$

car toute $\eta / 2$-presque période du système $F$ est évidemment une $\eta$-presque période de la fonction $f_{1}(x)+f_{2}(x)$.

Théor ème II. F étant un système de deux fonctions périodiques continues $f_{1}(x), f_{2}(x)$ dont les fréquences sont incommensurables et dont on connait les périodes $\pi_{1}, \pi_{2}$ et le module de continuité uniforme $\delta$ de l'une d'elles pour le nombre $\eta$, la oaleur de $L(F, \eta)$ admet l'estimation par la formule

$$
L(F, \eta) \leqslant \frac{2 q_{i_{0}}+1}{r} \pi_{1} \cdot \pi_{2}
$$

où $q_{i_{0}}$ et $r$ sont déterminés par un segment (dépendant de $\left.\delta\right) d u$ déoeloppement de $\pi_{1} / \pi_{2}$ en fraction continue.

Bien entendu, on peut y remplacer les modules de continuité par des nombres plus petits quelconques.

Démonstration. Admettons qué c'est la fonction $f_{2}(x)$ dont $\delta$ est le module de continuité uniforme pour la valeur donnée $\eta>0$. Posons $\beta=\delta / \pi_{2}$.

15) On appelle fréquence des nombres naturels $k$ appartenant à un ensemble $S$ le nombre $\lim _{N \rightarrow \infty} \frac{N^{\prime}}{N}$ où $N^{\prime}$ désigne le nombre des $k \in S$ qui ne dépassent pas $N$ et $N=1,2, \ldots$ 
Si nous arrivons à nommer une suite croissante de $\eta$-presque périodes communes des fonctions $f_{1}(x)$ et $f_{2}(x)$ - y compris 0 où la distance entre les termes consécutifs soit bornée par un où la distance nous aurons établi par cela-même l'existence de la grandeur (finie) $L(F, \eta)$, car tout segment de longueur $d$ contiendra grandeur (finie $L(F, \eta)$ cariodes communes en question. Toute estialors des $\eta$-presque périodes communes en question. To mation de la distance entre les termes d'une telle suite sera a fortiori une estimation de $L(F, \eta)$.

Or, l'ensemble des $\eta$-presque périodes communes des fonctions $f_{1}(x)$ et $f_{2}(x)$ contient évidemment tous les nombres de la forme $k \pi_{1}$ où $k$ est un nombre naturel pour lequel il existe un entier $p \geqslant 0$ tel que $\left|k \frac{\pi_{1}}{\pi_{2}}-p\right|<\beta$, c'est-à-dire tel que l'on a l'une des deux relations:

$$
\begin{gathered}
0<k \frac{\pi_{1}}{\pi_{2}}-p<\beta, \\
1-\beta<k \frac{\pi_{1}}{\pi_{2}}-(p-1)<1 .
\end{gathered}
$$

En effet, on a alors d'une part $\left|k \pi_{1}-p \pi_{2}\right|<\delta$, d'où $\left|f_{2}(x)-f_{2}(x+k \pi)\right|<\eta$ et d'autre part évidemment

$$
\left|f_{1}(x)-f_{1}\left(x+k \pi_{1}\right)\right|=0 .
$$

Désignons par $S^{\prime}(\beta)$ et $S^{\prime \prime}(\beta)$ respectivement les ensembles des $k$ qui satisfont à (i) et (ii) respectivement pour certains entiers $p \geqslant 0$. La fréquence des $k \in S^{\prime}(\beta)$, de même que celle des $k \in S^{\prime \prime}(\beta)$, est égale à $\beta$ en vertu du théorème de $H$. Weys.

Commençons par l'estimation de $L(F, \eta)$ dans le cas particulier où il existe un $q$ naturel pour lequel

$$
\beta=R\left(q \frac{\pi_{1}}{\pi_{2}}\right) \text {. }
$$

On a alors en vertu du théorème de Hecke-Ostrowski

$$
\left|N^{\prime}-N \cdot \beta\right| \leqslant q \quad \text { et } \quad\left|N^{\prime \prime}-N \cdot \beta\right| \leqslant q .
$$

où $N^{\prime}$ et $N^{\prime \prime}$ désignent respectivement les nombres des $k \leqslant N$ naturels qui appartiennent à $S^{\prime}(\beta)$ et à $S^{\prime \prime}(\beta)$.

L'estimation de la valeur absolue de la différence entre les nombres consécutifs appartenant à l'un des ensembles $S^{\prime}(\beta)$ et $S^{\prime \prime}(\beta)$ à l'ensemble $S^{\prime}(\beta)$ par exemple - sera basée sur le lemme suivant:
Si les nombres naturels d'un ensemble $S$ de tels nombres ont la fréquence $\beta$ et si l'écart absolu $\left|N^{\prime}-\beta N\right|$, où $N^{\prime}$ est le nombre des $k \leqslant N$ dans $S$, ne dépasse pas un entier fixe $q$, la distance entre les éléments ooisins de $S$ ne dépasse pas $(2 q+1) \mid \beta$.

Remarquons que la réciproque est fausse car la distance entre les termes voisins d'une suite croissante de nombres naturels admettant une fréquence peut être bornée sans que l'écart en question le soit.

La démonstration du lemme est simple. Considérons deux éléments voisins $k_{1}$ et $k_{2}$ de l'ensemble $S$ et désignons par $k_{1}^{\prime}$ et $k_{2}^{\prime}$ les nombres d'éléments de $S$ qui ne dépassent pas $k_{1}$ et $k_{2}$ respectivement. Posons

$$
k_{2}-k_{1}=d \quad \text { et } \quad k_{1}^{\prime}=k_{1} \beta+\gamma .
$$

On a donc conformément à l'hypothèse

$$
\begin{gathered}
|\gamma|<q, \quad k_{2}^{\prime}=k_{1}^{\prime}+1, \\
\beta\left(k_{1}+d\right)-q \leqslant k_{2}^{\prime} \leqslant \beta\left(k_{1}+d\right)+q, \\
\beta\left(k_{1}+d\right)-q \leqslant \beta k_{1}+\gamma+1 \leqslant \beta\left(k_{1}+d\right)+q .
\end{gathered}
$$

La dernière relation entraîne $\beta d-q \leqslant \gamma+1$, d'où

$$
d \leqslant(\gamma+1+q) / \beta \leqslant(2 q+1) / \beta,
$$

Ajoutons que $k_{0}$ désignant le plus petit élément de $S$, on a $k_{0} \beta-1<q$, d'où $k_{0}<(q+1) \beta$, de sorte que la thèse du lemme est satisfaite non seulement par la distance entre éléments voisins de $S$, mais aussi par celle entre 0 et son premier élément.

Le lemme établi, reprenons la démonstration du théorème dans le cas particulier où il existe un $q$ naturel assujetti à l'hypothèse supplémentaire (19). On conclut de (20) en vertu du lemme que la distance entre éléments voisins de l'ensemble $S^{\prime}$ ne dépasse pas $\frac{2 q}{\delta(\eta)} \pi_{i 2}$, ce qui entraîne aussitôt l'inégalité

$$
L(F, \eta) \leqslant \frac{2 q+1}{\delta(\eta)} \pi_{1} \cdot \pi_{2}
$$

Reste à envisager le cas général où, la restriction (19) étant levée, les nombres positifs $\eta$ et $\beta$ sont arbitraires. L'estimation de $L(F, \eta)$ peut alors être obtenue au moyen de l'algorithme d'Eu- 
clide, en développant le quotient $\pi_{1} / \pi_{2}$ en fraction continue régulière. En désignant par $p_{i} / q_{i}$ où $i=1,2, \ldots$ sa $i$-ème réduite, on a - comme on sait - l'inégalité

$$
\left|\frac{\pi_{1}}{\pi_{2}}-\frac{p_{i}}{q_{i}}\right|<\frac{1}{q_{i}^{2}}, \quad \text { d'où } \quad\left|\frac{\pi_{1}}{\pi_{2}} q_{i}-p_{i}\right|<\frac{1}{q_{i}} .
$$

Soit $i_{0}$ le premier indice tel que $q_{i}>\frac{1}{\beta}$. Donc $\left|\frac{\pi_{1}}{\pi_{2}} q_{i_{0}}-p_{i_{0}}\right|<\frac{1}{q_{i_{0}}}$ et par conséquent

(21) $\quad R\left(q_{i_{0}} \frac{\pi_{1}}{\pi_{2}}\right) \cdot \pi_{2}<\delta(\eta)$ ou bien $\left[1-R\left(q_{i_{0}} \frac{\pi_{1}}{\pi_{2}}\right)\right] \cdot \pi_{2}<\delta(\eta)$.

Le problème se réduit de cette manière au cas particulier envisagé, en posant respectivement

$$
r=R\left(q_{i_{0}} \frac{\pi_{1}}{\pi_{2}}\right) \cdot \pi_{2} \quad \text { ou bien } \quad r=\left[1-R\left(q_{i_{0}} \frac{\pi_{1}}{\pi_{2}}\right)\right] \cdot \pi_{2}
$$

et en remplaçant $\beta$ par $r / \pi_{2}$. En effet, tous les nombres $k / \pi_{1}$ pour lesquels on a $\left|k \frac{\pi_{1}}{\pi_{2}}-p\right|<\frac{r}{\pi_{2}}<\frac{\delta(\eta)}{\pi_{2}}$ avec un $p$ naturel sont des $\eta$-presque périodes communes de $f_{1}(x)$ et $f_{2}(x)$, et la distance de ces nombres ne dépasse pas $\frac{2 q_{i_{0}}+1}{r} \pi_{1} \cdot \pi_{2}$ en vertu de (21), du théorème de Hecke-Ostrowski et du lemme. On a par conséquent la formule (18), c. q. f. d.

Il est à remarquer que l'indice $i_{0}$ employé dans la démonstration du théorème II est relativement petit, car les dénominateurs des réduites croissent rapidement, en tout cas pas moins rapidement que les termes de la suite de Fibonacci, donc que $\left(\frac{\sqrt{5}+1}{2}\right)^{n}$.

Thé or ème III. F étant un système de deux fonctions périodiques continues $f_{1}(x)$ et $f_{2}(x)$ de périodes $\pi_{1}$ et $\pi_{2}$ et de modules de continuité uniforme $\delta_{1}(\eta)$ et $\delta_{2}(\eta)$ respectivement, donnés pour un $\eta>0$, si les dénominateurs $b_{1}, b_{2}, \ldots$ de la fraction continue

$$
\frac{\pi_{1}}{\pi_{2}}=b_{0}+\frac{1 \mid}{\mid b_{1}}+\frac{1 \mid}{\mid b_{2}}+\ldots
$$

ne dépassent pas $M$, on a l'estimation suivante:

(23) $L(F, \eta) \leqslant(M+2) \cdot \pi_{1} \cdot \pi_{2} \cdot \min \left\{\frac{1}{\delta_{1}(\eta)}\left[2 \frac{\pi_{1}}{\delta_{1}(\eta)}+1\right], \frac{1}{\delta_{2}(\eta)}\left[2 \frac{\pi_{2}}{\delta_{2}(\eta)}+1\right]\right\}$.
Remarquons que l'hypothèse de ce théorème est satisfaite en particulier lorsque $\pi_{1} / \pi_{2}$ est un nombre irrationnel algébrique du $2^{\text {me }}$ degré, car son développement en fraction continue est alors périodique.

Démonstration. En désignant par $p_{i} / q_{i}$ la $i$-ème réduite de (22), on a $q_{1}=b_{1}, q_{2}=b_{1} b_{2}+1$ et de façon générale $q_{i+1}=b_{i} q_{i}+q_{i-1}$, d'où par hypothèse

$$
q_{i+1} \leqslant M q_{i}+q_{i-1}
$$

pour $i=1,2, \ldots$

Posons $\beta=\delta_{2}(\eta) / \pi_{2}$. Si $\beta \geqslant 1 /\left(b_{1} b_{2}+1\right)$, c'est-à-dire si $\beta \geqslant 1 / q_{2}$, l'algorithme employé dans la démonstration du théorème II conduit aussitôt au but.

Admettons donc que $\beta<1 /\left(b_{1} b_{2}+1\right)$. Il existe alors un indice $i_{0}$ tel que

(25) $\quad q_{i_{j}-1}<\frac{1}{\beta}, \quad q_{i_{0}}<\frac{1}{\beta}, \quad q_{i_{0}+1} \geqslant \frac{1}{\beta}, \quad q_{i_{0}+2}>\frac{1}{\beta}, \quad \ldots$

Il en résulte en vertu de (24) que

$$
\frac{1}{q_{i_{0}}+q_{i_{0}+1}}>\frac{\beta}{M+2} \text {. }
$$

On sait de la théorie des fractions continues que

d'où

$$
1 / q_{i}\left(q_{i}+q_{i+1}\right)<\left|\pi_{1} / \pi_{2}-p_{i} / q_{i}\right|<1 / q_{i} q_{i+1},
$$

$$
\frac{1}{q_{i_{0}}+q_{i_{0}+1}^{\prime}}<\left|q_{i_{0}} \frac{\pi_{1}}{\pi_{2}}-p_{i_{0}}\right|<\frac{1}{q_{i_{0}+1}} \leqslant \beta
$$

ce qui entraîne en vertu de (26)

$$
\frac{\beta}{M+2}<\left|q_{i_{0}} \frac{\pi_{1}}{\pi_{2}}-p_{i_{0}}\right|<\beta
$$

On en conclut en vertu de (25) et du lemme (voir p. 135) que

$$
L(F, \eta) \leqslant \frac{2 q_{i_{0}}+1}{\left|q_{i_{0}} \frac{\pi_{1}}{\pi_{2}}-p_{i_{0}}\right|} \pi_{1}<\frac{2 \frac{\pi_{2}}{\delta_{2}(\eta)}+1}{\delta_{2}(\eta)}-(M+2) \cdot \pi_{1} \cdot \pi_{2} .
$$

Comme le raisonnement reste valable si l'on interchange les indices 1 et 2 , le théorème III se trouve établi. 
L'application des théorèmes II et III permet dans certains cas d'arriver à une estimation effective des $\varepsilon$-bases statistiques pour la fonction $f(x)=f_{1}(x)+f_{2}(x)$ au moyen du théorème I et pour le système des fonctions $f_{1}(x)$ et $f_{2}(x)$ au moyen du théorème $I^{\prime}$.

A savoir, suivant que les deux fonctions en question satisfont seulement aux hypothèses du théorème II ou aussi à celles du théorème III, on aura à substituer à $\eta$ dans la formule (18) ou (23) respectivement $1 / 2 \delta_{Q_{1}}\left(e^{2} / 5\right)$ ou $1 / 2 \delta_{Q_{2}}(\varepsilon / 5)$ pour employer le théorème I (voir exemple 3, p. 132, et remarque, p. 133), et $\min _{i=1} \delta_{g_{i}}\left({ }^{8} / 10\right)$ pour employer le théorème $l^{\prime}$.

Cette application du théorème II ou III exige donc l'estimation préalable de l'un des nombres

où $i, j=1,2$.

$$
\delta_{f_{i}}\left[1 / 2 \delta_{\rho_{j}}(\varepsilon / 5)\right], \quad \delta_{f_{i}}\left[\delta_{\rho_{j}}(\varepsilon / 10)\right],
$$

Ainsi par exemple, pour la fonction $f(x)=A \sin \lambda x$, on a $\delta_{e}(\varepsilon / 10)=2 A \sin ^{2}(\varepsilon \pi / 20)$ d'après (15). Comme le $\eta$-module de continuité uniforme de la même fonction $f(x)$ est

il vient

$$
\delta_{f}(\eta)=\frac{1}{\lambda} \arcsin (\eta / A)
$$

$$
\delta_{f}\left[\delta_{e}(\varepsilon / 10)\right]=\frac{1}{\lambda} \arcsin \left[2 \sin ^{2}(\varepsilon \pi / 20)\right]
$$

(ce module est donc indépendant de l'amplitude).

5. Considérons, pour terminer, quelques problèmes qui s'imposent.

Les théorèmes II et III constituent par eux-mêmes une preuve que la somme de deux fonctions périodiques satisfait toujours à la condition de la définition d'une fonction presque périodique de H. Bohr. Or, comme le montre M. Bohr (loco cit.), cette condition se transmet des sommandes à la somme. Sa démonstration est élémentaire et ne fait pas appel aux théorèmes de la théorie des nombres, mais il semble fort difficile d'en déduire une estimation de la grandeur $L(F, \eta)$, même dans le cas le plus simple de la somme de deux fonctions périodiques (qui a été envisagé plus haut).
Il semble que la grandeur en question est liée par sa nature à la théorie de l'équipartition. Cependant les théorèmes capitaux de cette théorie, même les plus connus (comme avant tout celui de H. WeYL par exemple), se montrent impuissants de fournir une estimation satisfaisante de $L(F, \eta)$ ou une démonstration d'existence de cette grandeur (finie), même dans le cas du système $F$ de deux fonctions périodiques, leurs thèses ne concernant que l'équipartition asymptotique.

Il est donc naturel de se demander si le théorème de НвскE, dont la nature est asser particulière, constitue en effet un élément indispensable de la démonstration en question.

Puis, la question se pose de trouver une estimation de $L(F, \eta)$ pour les systèmes $F$ de $m>2$ fonctions périodiques - et, enfin, pour une fonction presque périodique arbitraire - en relation avec leurs fréquences.

Une généralisation convenable du théorème de HeCKE-Ostrowski à l'équipartition simultanée d'un système de nombres ${ }^{16}$ ) pourrait peut-être contribuer à l'étude de ces questions.

16) Cf. le problème posé par l'auteur, Colloquium Mathematicum I.5 (1948), p. 239, P 37.

(Reçu par la Rédaction le 2. 6. 1948). 